\title{
Global Stability of Systems with Distributed and Concentrated Parameters
}

\author{
$\square^{1}$ Bulat Faritovich Bairamov, ${ }^{2}$ Farit Davletovich Bairamov \\ ${ }^{1,2}$ Department of Mechanics and Design, Automotive Department, Naberezhnye Chelny Institute (Branch), KFU \\ bbairamov@gmail.com \\ FDBajramov@kpfu.ru
}

Received: 21st August 2020, Accepted: 14th September 2020, Published: 31st October 2020

\begin{abstract}
The paper considers the problem of stability in the systems with distributed and lumped parameters defined by linear differential equations partially and ordinary derivatives. The issue is resolved through the process of Lyapunov functions (functionals). The major problem of the method used is the development of the corresponding Lyapunov procedures. In most applications, such functionals were regularly formed intuitively, based on the first integrals, whole energy, and other factors. to make the solution of this problem more manageable, it has been recommended to modify the original higher-order differential equations into a system of first-order equations. When the order of higherorder partial differential equations is reduced by the introduction of additional variables for derivatives concerning spatial coordinates, the equations without time derivatives appear. To take such equations into account when they calculate the derivative of the Lyapunov function in view of the system under consideration, a modified method of Lagrange multipliers is used. The transition to partial differential equations of the first order collectively with the writing of ordinary differential equations in the standard Cauchy form provides to use specific equations appropriately to develop the Lyapunov function as a total of integral and regular quadratic patterns, the sign-definiteness of which is checked by the Sylvester criterion, and to create a universal method to study stability in general for a broad class of systems with distributed and lumped parameters. The conditions for asymptotic stability, in general, are obtained. The results obtained in the article make it possible to study various complex engineering objects with distributed and lumped parameters. As an example, they take into account the stability of a rotary-type wind turbine operation with a load (pump and generator) and the elasticity of the shaft transmitting torque from the wind turbine to the load. The use of environmentally friendly wind turbines that can decrease energy expenses is an assuring area. A rotary-type wind turbine does not require a wind orientation system, it is safe, quiet, and contrary to propeller wind turbines can be installed near buildings and structures.
\end{abstract}

Keywords

The Systems with Distributed and Lumped Parameters; Stability in General; The Method of Lyapunov Functions; Quadratic Forms

\section{Introduction}

The emergence of the concept of stability as a whole, as the generalization of Lyapunov's stability, is associated with technical considerations. Since in many technical problems, it is essential that the unperturbed motion be asymptotically stable, and this stability takes place for any, even arbitrarily large, initial perturbations. In a number of problems, along with arbitrary initial ones, it is also required to take into account finite permanent perturbations (FPP). This led to the emergence of the concept of asymptotic stability in the whole under FPP. It should be noted that when FPP is taken into account, the perturbed trajectories will approach asymptotically not the unperturbed trajectory itself, but only some of its vicinity.

One of the main methods for studying the stability of the systems with distributed parameters is the method of Lyapunov functions (functionals). At the same time, along with purely distributed systems, the systems with distributed and lumped parameters were also considered. A fairly complete overview and state of the art in this area can be found in $[7 ; 9 ; 10 ; 2]$. Along with theoretical studies by the method of Lyapunov functions, the studies of specific objects with distributed parameters were carried out. For example, elastic and aeroelastic objects $[2 ; 6 ; 8 ; 1]$, chemical reactors [7], liquid-propellant rocket engines [7;2], etc. [7;2]. In applications, the main difficulty is the construction of the corresponding Lyapunov functionals, which, during the study of specific objects, were usually constructed intuitively, proceeding from the total energy, first integrals, and other considerations. In the works [2;3], when they solve stability problems for the systems with distributed parameters, it is proposed to reduce the initial high-order partial differential equations to the system of first-order equations in time and spatial coordinates. Further, for this system, Lyapunov functionals are constructed from specific equations in the form of integral quadratic forms, 
the definiteness of signs of which can be checked using the well-known Sylvester criterion. This approach allows to develop Lyapunov functional constructively and expands the possibilities of Lyapunov function method use in specific applications significantly.

In this paper, the idea of transforming the original higher-order equations into a system of first-order equations is used to study the stability of the systems with distributed and lumped parameters.

\section{Methods}

The work uses mathematically rigorous and precise research methods: the methods of the theory of partial differential equations, the method of Lyapunov functions, the method of Lagrange multipliers, and matrix theory.

When they calculate the derivative of the Lyapunov function (2.1) by virtue of equations (1.1) - (1.3), an approach is used to take into account the second equation (1.1) without time derivatives, which is similar in its procedure to the Lagrange multiplier method.

Stability conditions are written on the basis of well-known classical results of finite-dimensional and distributed system stability theory. The sign-definiteness of ordinary and integral quadratic forms is checked by the Sylvester criterion.

\section{Results and Discussion}

\section{Problem Statement}

Let's consider the system with one distributed and other finite-dimensional links, the perturbed state of which is described by the following equations:

$$
\begin{aligned}
& \frac{\partial \varphi}{\partial t}=A(x) \frac{\partial \varphi}{\partial x}+B(x) \frac{\partial \psi}{\partial x}+A_{0}(x) \varphi+B_{0}(x) \psi \\
& C(x) \frac{\partial \varphi}{\partial x}+D(x) \frac{\partial \psi}{\partial x}+C_{0}(x) \varphi+D_{0}(x) \psi=0 \\
& x \in(0,1) \\
& \frac{d z}{d t}=F_{1} z+F_{2} \varphi(0, t)+F_{3} \varphi(1, t), \\
& \Gamma_{1} \varphi(0, t)=\Gamma_{2} z, \quad \Gamma_{3} \varphi(1, t)=\Gamma_{4} z \\
& t \in I=(0, \infty),
\end{aligned}
$$

where $\varphi=\varphi(x, t)-n-$ dimensional vector of the state variables of the distributed link, $\psi=\psi(x, t)-\ell-$ dimensional vector of the state variables of this link, the time derivatives of which are not included in the system (1.1), $z=z(t)-m-$ dimensional vector of the state variables of the finite-dimensional links, $A(x), B(x), C(x)$, $D(x), A_{0}(x), B_{0}(x), C_{0}(x), D_{0}(x)$ - the matrices whose elements are bounded continuous functions, $F_{1}$, $F_{2}, F_{3}, \Gamma_{1}, \Gamma_{2}, \Gamma_{3}, \Gamma_{4}$ - constant matrices.

From the mathematical point of view, the problem (1.1) - (1.3) is a boundary value problem for partial differential equations. The equations (1.1) represent the general form of writing any linear partial differential equation of arbitrary order in the form of the first order partial differential equations [2;3]. To transform higher-order equations to the form (1.1), one should take the lowest derivatives as additional variables and write down the original equation and the integrability condition in these variables.

The equations (1.3) are simple boundary conditions connecting the boundary values of the components $\varphi(x, t)$ with each other or with the variable $Z$. The equation of dynamics (1.2) of finite-dimensional links located at both ends of the distributed link contains the boundary values $\varphi(x, t)$ and represents a complex boundary condition in the form of a differential equation.

The equations of the type (1.1) - (1.3) describe the systems that have elastic shafts of considerable length, for example, between the engine and the working machine (generator, pump, compressor, etc.); the systems containing pipelines (mains), in which it is necessary to take into account the distributed nature of liquid or gas flow, etc. 
Let's introduce the following measure:

$$
\rho=\int_{0}^{1} \varphi^{T} \varphi d x
$$

characterizing the disturbed state of the distributed link.

\section{Definition 1}

The system (1.1) - (1.3) is called asymptotically stable in the large with respect to the variables $\rho, z$, if it is stable in small by these variables and all solutions of the system (1.1) - (1.3) with arbitrarily large initial data remain bounded and $\lim \rho=0, \lim z=0$ at $t \rightarrow \infty$.

Let's consider the stability of the system (1.1) - (1.3) in general by the variables $\rho, z$.

1. Stability study. Let's consider the Lyapunov function to solve this problem.

$$
V=V_{1}+V_{2}=\int_{0}^{1} \varphi^{T}(x, t) v(x) \varphi(x, t) d x+z^{T}(t) Q z(t),
$$

where $v(x), Q$ - symmetric matrices: the elements $Q$ are constants, and the elements $v(x)$ - continuous bounded functions.

A feature of the system (1.1) - (1.3) is that the second equation (1.1) and the equations (1.3) do not contain time $t$ derivatives. This does not allow to calculate the derivative $V$ directly due to the entire system. First, we calculate the derivative $d V / d t$ due to the first equation (1.1) and the equation (1.2):

$$
\begin{aligned}
& \frac{d V}{d t}=\int_{0}^{1}\left[\varphi^{T} v\left(A \frac{\partial \varphi}{\partial x}+B \frac{\partial \psi}{\partial x}\right)+\left(\frac{\partial \varphi^{T}}{\partial x} A^{T}+\frac{\partial \psi^{T}}{\partial x} B^{T}\right) v \varphi+\right. \\
& \left.+\varphi^{T}\left(v A_{0}+A_{0}^{T} v\right) \varphi+\varphi^{T} v B_{0} \psi+\psi^{T} B_{0}^{T} v \varphi\right] d x+ \\
& +z^{T}\left(Q F_{1}+F_{1}^{T} Q\right) z+2 \varphi^{T}(0, t) F_{2}^{T} Q z+2 \varphi^{T}(1, t) F_{3}^{T} Q z .
\end{aligned}
$$

Following the Lagrange multiplier method, we add to this derivative the following expression to take into account the second equations (1.1):

$$
\begin{aligned}
& \int_{0}^{1}\left[\left(\varphi^{T} P_{1}+\psi^{T} P_{2}\right)\left(C \frac{\partial \varphi}{\partial x}+D \frac{\partial \psi}{\partial x}+C_{0} \varphi+D_{0} \psi\right)+\right. \\
& \left.+\left(\frac{\partial \varphi^{T}}{\partial x} C^{T}+\frac{\partial \psi^{T}}{\partial x} D^{T}+\varphi^{T} C_{0}^{T}+\psi^{T} D_{0}^{T}\right)\left(P_{1}^{T} \varphi+P_{2}^{T} \psi\right)\right] d x=0,
\end{aligned}
$$

where $P_{1}=P_{1}(x), P_{2}=P_{2}(x)$ - are arbitrary matrices for a while. The brackets $\left(\varphi^{T} P_{1}+\psi^{T} P_{2}\right)$, $\left(P_{1}^{T} \varphi+P_{2}^{T} \psi\right)$ play the role of Lagrange multipliers.

Let us perform integration by parts and require that the matrices $v, P_{1}, P_{2}, Q$ satisfy the following conditions:

$$
\begin{aligned}
& v A+P_{1} C=A^{T} v+C^{T} P_{1}^{T}, \quad P_{2} D=D^{T} P_{2}^{T}, \\
& v B+P_{1} D=C^{T} P_{2}^{T}, \quad P_{2} D_{0}+D_{0}^{T} P_{2}^{T}=\frac{d P_{2} D}{d x}, \\
& v B_{0}+P_{1} D_{0}+C_{0}^{T} P_{2}^{T}=\frac{d\left(v B+P_{1} D\right)}{d x}, \quad x \in(0,1) .
\end{aligned}
$$

and the following boundary conditions: 


$$
\begin{aligned}
& P_{2}(0) D(0)=P_{2}(1) D(1)=v(0) B(0)+P_{1}(0) D(0)= \\
& =v(1) B(1)+P_{1}(1) D(1)=0 .
\end{aligned}
$$

Then we get the following for the derivative $d V / d t$ :

$$
\begin{aligned}
& \frac{d V}{d t}=-\int_{0}^{1} \varphi^{T} w \varphi d x+z^{T}\left(Q F_{1}+F_{1}^{T} Q\right) z+2 \varphi^{T}(1) F_{3}^{T} Q z+2 \varphi^{T}(0) F_{2}^{T} Q z+ \\
& +\varphi^{T}(1) M(1) \varphi(1)-\varphi^{T}(0) M(0) \varphi(0),
\end{aligned}
$$

where

$$
\begin{aligned}
& w(x)=\frac{d M(x)}{d x}-v A_{0}-A_{0}^{T} v-P_{1} C_{0}-C_{0}^{T} P_{1}^{T}, \\
& M(x)=v A+P_{1} C .
\end{aligned}
$$

By virtue of boundary conditions (1.3), the following relations are true:

$$
\begin{aligned}
& \varphi(1)=E \varphi(1)=\Lambda_{1} \varphi(1)+\Gamma_{2} z, \\
& \varphi^{T}(1)=\varphi^{T}(1) \Lambda_{1}^{T}+z^{T} \Gamma_{2}^{T}, \\
& \varphi(0)=\Lambda_{2} \varphi(0)+\Gamma_{4} z, \\
& \varphi^{T}(0)=\varphi^{T}(0) \Lambda_{2}^{T}+z^{T} \Gamma_{4}^{T},
\end{aligned}
$$

where $\Lambda_{1}=E-\Gamma_{1}, \Lambda_{2}=E-\Gamma_{3}, E$ - a single matrix.

Substituting these relations into the last two terms (2.4) and requiring that the matrices $v, Q$ satisfy the following conditions additionally:

$$
\begin{aligned}
& F_{3}^{T} Q+\Lambda_{1}^{T} M(1) \Gamma_{2}=F_{2}^{T} Q-\Lambda_{2}^{T} M(0) \Gamma_{4}=0, \\
& \Lambda_{1}^{T} M(1) \Lambda_{1}=\Lambda_{2}^{T} M(0) \Lambda_{2}=0,
\end{aligned}
$$

the derivative (2.4) is transformed to the following form:

$$
\frac{d V}{d t}=-\int_{0}^{1} \varphi^{T} w \varphi d x-z^{T} \omega z,
$$

where

$$
\omega=-\left(Q F_{1}+F_{1}^{T} Q\right)-\Gamma_{2}^{T} M(1) \Gamma_{2}+\Gamma_{4}^{T} M(0) \Gamma_{4} .
$$

The equations (2.5) - (2.7), (2.9) can be used to develop the matrices $v(x), Q$ at given matrices $w(x), \omega$.

According to the well-known results on the stability of distributed [7] and finite-dimensional systems [5], the system (1.1) - (1.3) will be asymptotically stable in general with respect to the variables $\rho, z$, if, in an arbitrarily large region of the unperturbed process $\varphi=\psi=z=0$ :

a) the functional $V_{1}$ is continuous and definitely positive in measure $\rho$ and the following conditions are satisfied:

$$
\lim V_{1}=\infty \text { at } \rho \rightarrow \infty \text { and } \lim \rho[\varphi]=\infty \text { at } V_{1} \rightarrow \infty
$$

б) the function $V_{2}$ is definitely positive;

в) the derivative $d V / d t$ by virtue of the system (1.1) - (1.3) is definitely negative with respect to the variables $\rho$ , $z$ at $t \in I$.

Let the matrix $v(x)$ be definitely positive at $x \in(0,1)$. Then the integral form $V_{1}$ taking into account the 
boundedness of the matrix $v(x)$ satisfies the following conditions:

$$
\lambda_{1} \rho \leq V_{1} \leq \lambda_{1} \rho, \lambda_{1}, \lambda_{2}-\text { const }>0,
$$

i.e. is definitely positive and continuous in measure $\rho$. Here

$$
\lambda_{1}=\inf _{x \in(0,1)} \lambda_{\min }(x), \lambda_{2}=\sup _{x \in(0,1)} \lambda_{\max }(x),
$$

$\lambda_{\min }(x), \lambda_{\max }(x)$ - the minimum and maximum eigenvalues of the matrix $v(x)$, respectively. The condition a) is satisfied.

Suppose the matrix $\mathrm{Q}$ is also definitely positive. Then the form $V_{2}$ and, due to the boundedness of the matrix $\mathrm{Q}$, satisfies the inequalities [2]:

$$
\mu_{1}\|z\|^{2} \leq V_{2} \leq \mu_{2}\|z\|^{2}, \mu_{1}, \mu_{2}-\text { const }>0
$$

where $\mu_{1}, \mu_{2}$ - the minimum and maximum eigenvalues of the matrix $\mathrm{Q}$, respectively. The condition $\mathrm{b}$ ) is also fulfilled.

The derivative (2.8) will be definitely negative in the variables $\rho, z$, if the matrix $w(x)$ is definitely positive at $x \in(0,1)$, and the matrix $\omega$ is definitely positive.

The continuity of the integral form $V_{1}$ in measure $\rho$ directly follows from the boundedness of the matrix $v(x)$ elements. The remaining conditions of the above propositions a), б), в) will be satisfied if the matrices $Q$ and $\omega$ are definitely positive, and $v(x)$ and $w(x)$ are definitely positive at $x \in(0,1)$, i.e.

$$
Q>0, \quad \omega>0, \quad v(x)>0, \quad w(x)>0, \quad x \in(0,1) .
$$

\section{Example}

Let us consider the stability of a rotary wind turbine operation with a vertical axis of rotation [4] together with the load (generator, pump, etc.). The shaft that transmits the torque of the wind turbine to the load has a considerable length, so the problem is solved, taking into account the elasticity of this shaft.

Note that the use of environmentally friendly wind turbines that reduce energy costs is a promising area. The rotary-type wind turbine does not require a wind orientation system, is quiet, safe and, unlike propeller wind turbines, can be placed near settlements and buildings.

The equations of a wind turbine dynamics with a load and with an elastic transmission shaft obtained earlier in [4], will be written with an additional account of the transmission shaft end connection elasticity with the load shaft in relative deviations from the nominal operating mode of the unit.

$$
\begin{aligned}
& \frac{d z}{d t}=k z+\frac{\partial \varphi(x, t)}{\partial x}_{\mid x=0}, \\
& \frac{\partial^{2} \varphi(x, t)}{\partial t^{2}}=a \frac{\partial^{2} \varphi(x, t)}{\partial x^{2}}, \quad x \in(0,1),
\end{aligned}
$$

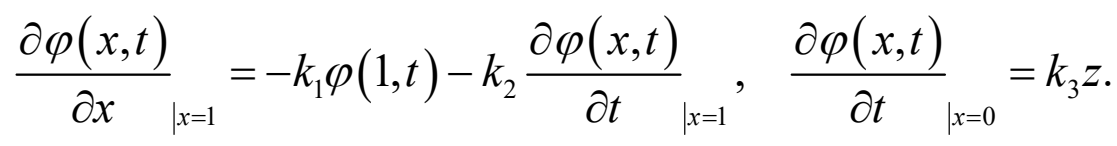

Here $x=\frac{y}{\ell}, z=\frac{\omega-\omega_{*}}{\omega_{*}}, \varphi(x, t)=\frac{\psi(x, t)-\psi_{*}(x, t)}{\psi_{\text {max }_{*}}}, \psi_{\max _{*}}=\frac{M_{*} \ell}{G I}, a=\frac{G I}{J \ell^{2}}, k=\frac{1}{J_{b}}\left(\frac{\partial M_{b}}{\partial \omega}\right)_{*}$, $k_{1}=\frac{\lambda}{G I}, k_{2}=\frac{\ell}{G I}\left(\frac{\partial M}{\partial \omega}\right)_{*}, k_{3}=\frac{G I \omega_{*}}{\ell M_{*}}, y-$ the coordinates of the cross-sections of the transmission shaft, $\omega-$ 
the angular velocity of the wind turbine, $\psi(x, t), \ell, J, G I$ - the absolute angle of section rotation, length, a linear moment of inertia, the torsional stiffness of the transmission shaft, $M_{b}, M-$ the torques of the wind turbine and load, $J_{b}$ - the moment of the wind turbine inertia, $\psi_{\text {max }_{*}}-$ the maximum angle of the transmission shaft twisting in the nominal mode, $\lambda$ - the coefficient of elasticity of the transmission shaft end in the load shaft, the sign $\left(^{*}\right)$ indicates the values in the nominal mode of the unit operation, when $\omega_{*}=$ const , $M_{b}=M=M_{*}=$ const and the transmission shaft has a constant length of static deformation $\partial \psi_{*} / \partial x=M_{*} / G I$.

Introducing new variables $\varphi_{1}=\varphi(x, t), \varphi_{2}=\partial \varphi / \partial t, \varphi_{3}=\partial \varphi / \partial x$ and taking into account the integrability condition $\partial \varphi_{3} / \partial t=\partial \varphi_{2} / \partial x$, let's put down the equations (3.1) in the form of the system (1.1) (1.3), where:

$$
\begin{aligned}
& A=\left\|\begin{array}{lll}
0 & 0 & 0 \\
0 & 0 & a \\
0 & 1 & 0
\end{array}\right\|, \quad A_{0}=\left\|\begin{array}{lll}
0 & 1 & 0 \\
0 & 0 & 0 \\
0 & 0 & 0
\end{array}\right\|, \quad C=\|1 \quad 0 \quad 0\|, \quad C_{0}=\left\|\begin{array}{ccc}
0 & 0 & -1
\end{array}\right\|, \\
& F_{1}=k, F_{2}=\left\|\begin{array}{lll}
0 & 0 & 1
\end{array}\right\|, \Gamma_{1}=\left\|\begin{array}{lll}
0 & 1 & 0 \\
0 & 0 & 0 \\
0 & 0 & 0
\end{array}\right\|, \Gamma_{2}=\left\|\begin{array}{ccc}
k_{3} \\
0 \\
0
\end{array}\right\|, \Gamma_{3}=\left\|\begin{array}{ccc}
k_{1} & k_{2} & 1 \\
0 & 0 & 0 \\
0 & 0 & 0
\end{array}\right\|,
\end{aligned}
$$

And the matrices $B, B_{0}, D, D_{0}, F_{3}, \Gamma_{4}$ are zero ones.

Let us develop the functional (2.1), where we take $V_{2}=q z^{2}, q=$ const $>0$ in this example. Equation (2.3) is performed automatically. Let us put down the equations (2.2), (2.5) in the scalar form:

$$
\begin{aligned}
& P_{21}=v_{13}, \quad P_{31}=a v_{12}, \quad v_{33}=a v_{22} . \\
& \frac{d P_{11}}{d x}=w_{11}, \quad \frac{d v_{23}}{d x}-2 v_{12}=w_{22}, \\
& a \frac{d v_{23}}{d x}+2 a v_{12}=w_{33}, \quad a \frac{d v_{22}}{d x}=w_{23}, \\
& a \frac{d v_{12}}{d x}+P_{11}=w_{13}, \quad \frac{d v_{13}}{d x}-v_{11}=w_{12},
\end{aligned}
$$

where $P_{i j}, v_{i j}, w_{i j}$ - the elements of the matrices $P, v, w$.

The equations (2.7) yield the following boundary conditions at $x=0$ and $x=1$ :

$$
P_{11}(0)=v_{23}(0)=v_{13}(0)=0, \quad q=a k_{3} v_{22}(0),
$$

a) $P_{11}(1)-2 a k_{1} v_{12}(1)-a k_{1}^{2} v_{23}(1)=0$,

б) $\left(1+a k_{2}^{2}\right) v_{23}(1)-2 a k_{2} v_{22}(1)=0$,

в) $v_{13}(1)-a k_{2} v_{12}(1)-k_{1}\left[v_{33}(1)-a k_{2} v_{23}(1)\right]=0$.

Let $w_{11}=$ const, $w_{22}=1, w_{33}=a, w_{13}=P_{11}, w_{12}=w_{23}=0$.

Solving the first five equations (3.3) with the boundary conditions (3.4), (3.5 a), (3.5 b), we obtain the following: 


$$
v_{22}=\frac{\left(1+a k_{2}^{2}\right)}{2 a k_{2}}, v_{12}=0, v_{23}=x, P_{11}=w_{11} x, w_{11}=P_{11}(1)=a k_{1}^{2}, w_{13}=a k_{1}^{2} x .
$$

From the last equations (3.2), (3.4) it follows that $v_{33}=a v_{22}, q=\frac{\left(1+a k_{2}^{2}\right) k_{3}}{2 k_{2}}$, and from (2.9) we find $\omega=-2 q k$

The functions $v_{11}$ and $v_{13}$ are included only in the last equation (3.3). Assuming that $v_{11}=$ const, from this equation and the boundary condition $(3.5 \mathrm{в})$, we find the following:

$$
v_{11}=\frac{k_{1}\left(1-a k_{2}^{2}\right)}{2 k_{2}}, \quad v_{13}=v_{11} x
$$

The functional $V$ (2.1) and its derivative $d V / d t$ (2.8) by virtue of the system (3.1) will be written in the following form:

$$
\begin{aligned}
& V=\int_{0}^{1}\left[\frac{k_{1}\left(1-a k_{2}^{2}\right)}{2 k_{2}}\left(\varphi_{1}^{2}+2 x \varphi_{1} \varphi_{3}\right)+\frac{1+a k_{2}^{2}}{2 a k_{2}}\left(\varphi_{2}^{2}+a \varphi_{3}^{2}\right)+2 x \varphi_{2} \varphi_{3}\right] d x+ \\
& +\frac{\left(1+a k_{2}^{2}\right) k_{3}}{2 k_{2}} z^{2} \\
& \frac{d V}{d t}=-\int_{0}^{1}\left[a k_{1}^{2}\left(\varphi_{1}^{2}+2 x \varphi_{1} \varphi_{3}\right)+\varphi_{2}^{2}+a \varphi_{3}^{2}\right] d x+\frac{k k_{3}\left(1+a k_{2}^{2}\right)}{k_{2}} z^{2} .
\end{aligned}
$$

Since $k_{1}>0, k_{3}>0$, then from inequalities (2.10) we find the conditions for the wind turbine asymptotic operation stability in general with a load and taking into account the elasticity of the transmission shaft and the expression for the coefficient $\mathrm{k}$ in the following form:

$$
\left(\frac{\partial M_{b}}{\partial \omega}\right)_{*}<0, \quad 0<k_{2}<\frac{1}{\sqrt{a}} .
$$

From the comparison of these conditions with the stability condition $\left(\frac{\partial M}{\partial \omega}\right)_{*}-\left(\frac{\partial M_{b}}{\partial \omega}\right)_{*}>0$ for a wind turbine with a load and with a rigid transmission shaft, it can be seen that the elasticity of the shaft narrows the stability region.

\section{Conclusions}

- Sufficient stability conditions have been obtained for the systems with distributed and lumped parameters in general, presented in the sort of inequalities relating the system's coefficients.

- The improved methodology for studying stability is quite effective and general. For instance, it enables analyzing the stability of a relatively broad range of objects with distributed and lumped parameters in general.

- The survey possesses functional and theoretical value.

\section{Summary}

The alteration of high-order partial differential equations into a system of first-order partial differential equations, collectively with the writing of ordinary differential equations in Cauchy normal form, permits to develop the Lyapunov function constructively as a total of integral and ordinary quadratic forms as well as to generate a common method to analyze the stability of the systems with distributed and lumped parameters in general.

\section{Acknowledgement}

The study has been carried out based on the Russian Government Program of Competitive Growth of Kazan University. 


\section{References}

[1] Bairamov, B.F., \& Bairamov, F.D. (2019). On the stability of a class of linear systems with distributed and lumped parameters. Mechanics of Solids, 54(2), 245-250.

[2] Bayramov, F.D. (1995). stability and optimal stabilization of the systems with distributed parameters. Moscow: Mashinostroenie, $160 \mathrm{p}$.

[3] Bayramov, F.D., Bayramov, B.F., Galimov, N.S., \& Fardeev, A.R. (2016). Technological processes with combined power supply: mechanics, control, automation. Kazan: The Publishing house of Kazan University, 342 p. [4] Bayramov, F.D., Bayramov, B.F., \& Mardamshin, I.G. (2009). Mathematical modelling and a hydraulic system stability with a wind pump unit”. Bulletin of KSTU named after A.N. Tupolev, Kazan, 4, 42-47.

[5] Malkin, I.G. (1966). theory of motion stability. Moscow: Nauka, $530 \mathrm{p}$.

[6] Parks, P.S. (1967). A stability criterion for a panel flutter problem via the second method of Liapunov. Differentials equations and dynamical systems, New-Jorn - London: Acad. Press, 287-298.

[7] Sirazetdinov, T.K. (1987). stability of the systems with distributed parameters. Novosibirsk: Nauka. Siberian branch, $231 \mathrm{p}$.

[8] Wang, P.K. (1965). Stability analysis of siplifed flexible vehicle via Liapunov's direct method. AIAA Journal, 3(9), 1764-1766.

[9] Wang, P.K.C. (1966) On the stability of equilibrium of mixed distributed and lumped parameter control systems. Int. J. Control, 3(2), 139-147.

[10] Wang, P.K.C. (1968). The theory of stability and control for distributed parameter systems. Int. J. Control, 7(2), 101-116, (Bibliography). 